\title{
Ostéonécroses en rapport avec un traitement par bisphosphonates : présentation d'une « Fiche de liaison » facilitant la prévention
}

\author{
Salino S, Bodard AG, Brantus JF \\ Centre Léon Bérard, Lyon, France \\ salino@lyon.fnclcc.fr
}

L'ostéonécrose des maxillaires (ONM) figure parmi les effets secondaires potentiels d'un traitement par bisphosphonates. Cette complication a fait l'objet de très nombreuses publications depuis sa première description en 2003 par Marx. Sa prise en charge est plus ou moins invasive en fonction du stade de l'ONM et selon les auteurs.

Les traitements à disposition actuellement, qu'ils soient conservateurs ou qu'ils privilégient une attitude interventionniste, ne donnent globalement pas de résultats probants, l'ONM continuant d'évoluer la plupart du temps. Ceci explique sans doute, au moins partiellement, l'absence de consensus quant aux modalités de prise en charge des ONM. Et faute de traitement curatif efficace et éprouvé, la plupart des publications récentes insistent sur l'importance d'une approche préventive des ONM.

Des études récentes ont démontré que le risque de déclencher une ONM diminue de façon significative parmi les patients pour lesquels des mesures préventives avaient été instaurées, comparés aux patients qui n'en avaient pas bénéficié. Parmi ces mesures figurent notamment un bilan et une mise en état bucco-dentaires avant l'instauration d'un traitement par BPs. Ceci implique une sensibilisation du médecin prescripteur et du chirurgien-dentiste à ce problème potentiel, ainsi qu'une étroite collaboration entre ces protagonistes pour que ces mesures préventives puissent être mises en œuvre au moment opportun.

Dans le cadre d'un groupe de travail sur l'élaboration d'un thésaurus concernant les traitements par BPs, une "Fiche de liaison » a été mise au point. Elle vise à faciliter la communication entre les différents acteurs de la prise en charge et à promouvoir la prévention des ONM. Elle se présente sous forme d’un triptyque conservé par le patient et présenté aux différents professionnels afin qu'ils y consignent les informations essentielles tant sur le plan du traitement par BPs que sur le plan bucco-dentaire.

L'objectif de l'exposé est de présenter cette « Fiche de liaison ». 